\title{
Endoscopic treatment or balloon-occluded retrograde transvenous obliteration is safe for patients with esophageal/gastric varices in Child-Pugh class C end-stage liver cirrhosis
}

Keiji Yokoyama, Ryo Yamauchi, Kumiko Shibata, Hiromi Fukuda, Hideo Kunimoto, Kazuhide Takata, Takashi Tanaka, Shinjiro Inomata, Daisuke Morihara, Yasuaki Takeyama, Satoshi Shakado, and Shotaro Sakisaka

Division of Gastroenterology and Hepatology, Department of Internal Medicine, Fukuoka University Faculty of Medicine, Fukuoka, Japan

Background/Aims: There is a controversy about the availability of invasive treatment for esophageal/gastric varices in patients with Child-Pugh class C (CP-C) end-stage liver cirrhosis (LC). We have evaluated the validity of invasive treatment with CP-C end-stage LC patients.

Methods: The study enrolled 51 patients with CP-C end-stage LC who had undergone invasive treatment. The treatment modalities included endoscopic variceal ligation in 22 patients, endoscopic injection sclerotherapy in 17 patients, and balloon-occluded retrograde transvenous obliteration (BRTO) in 12 patients. We have investigated the overall survival (OS) rates and risk factors that contributed to death within one year after treatment.

Results: The OS rate in all patients at one, three, and five years was $72.6 \%, 30.2 \%$, and $15.1 \%$, respectively. The OS rate in patients who received endoscopic treatment and the BRTO group at one, three, and five years was $67.6 \%, 28.2 \%$ and $14.1 \%$ and $90.0 \%, 36.0 \%$ and $18.0 \%$, respectively. The average of Child-Pugh scores (CPS) from before treatment to one month after variceal treatment significantly improved from 10.53 to $10.02(P=0.003)$. Three significant factors that contributed to death within one year after treatment included the presence of bleeding varices, high CPS ( $\geq 11)$, and high serum total bilirubin levels ( $\geq 4.0 \mathrm{mg} / \mathrm{dL}$ ).

Conclusions: The study demonstrated that patients with a CPS of up to 10 and less than $4.0 \mathrm{mg} / \mathrm{dL}$ of serum total bilirubin levels may not have a negative impact on prognosis after invasive treatment for esophageal/gastric varices despite their CP-C end-stage LC. (Clin Mol Hepatol 2019;25:183-189)

Keywords: Portal hypertension; Liver cirrhosis; Esophageal and gastric varices; Endoscopy; Balloon occlusion

\footnotetext{
Study Highlights

The study evaluated the validity of endoscopic treatment and balloon-occluded retrograde transvenous obliteration for esophageal/gastric varices in patients with Child-Pugh class C end-stage liver cirrhosis. The study demonstrated that patients with a Child-Pugh scores of up to 10 and less than $4.0 \mathrm{mg} / \mathrm{dL}$ of serum total bilirubin levels may not have experienced a negative impact on prognosis after invasive treatment.
}

\begin{abstract}
Abbreviations:
BRTO, balloon-occluded retrograde transvenous obliteration; CP-C, Child-Pugh class C; CPS, Child-Pugh scores; EIS, endoscopic injection sclerotherapy; EVL, endoscopic variceal ligation; HCC, hepatocellular carcinoma; LC, liver cirrhosis; OS, overall survival
\end{abstract}

\section{Corresponding author : Keiji Yokoyama}

Division of Gastroenterology and Hepatology, Department of Interna Medicine, Fukuoka University Faculty of Medicine, 7 Chome-45-1 Nanakuma, Jonan Ward, Fukuoka 814-0180, Japan

Tel: +81-92-801-1011 (Ext.3355), Fax +81-92-874-2663

E-mail:yokotin@fukuoka-u.ac.jp

https://orcid.org/0000-0003-1320-6545

Received : May 15, 2018/ Revised : Aug. 24, 2018/ Accepted : Sep. 11, 2018 


\section{INTRODUCTION}

Portal hypertension in advanced liver cirrhosis (LC) may be caused by various factors including obstruction of the terminal hepatic veins by regenerative nodules, fibrous proliferation of the perisinusoidal space, and sinusoidal narrowing due to hepatocyte degeneration, which increase intrahepatic venous pressure and inflow into the portal vein. ' Because portal vessels lack venous valves to prevent backflow of blood, a part of portal blood flow that is normally antegrade hepatopetal becomes retrograde hepatofugal, leading to the formation of collateral pathways. ${ }^{2-5}$ The growth of collateral pathways and local hyperdynamic state of the upper abdomen in portal hypertension can result in the development of esophageal/gastric varices. ${ }^{6}$ Rupture of esophageal/gastric varices may lead to life-threatening and massive bleeding. Therefore, patients with varices at risk of rupture should be treated prophylactically even in cases without bleeding.

It is generally recommended that invasive treatment of esophageal/gastric varices should devise treatment policies very carefully in patients with Child-Pugh class C (CP-C) end-stage LC. This population has a high risk of hepatic failure because of decreased intrahepatic blood flow or portal thrombosis after the procedure. ${ }^{7.8}$ However, although invasive treatment is often required in cases of hemorrhagic shock caused by ruptured varices, there is a lack of consensus on its effects.

In the present study we aimed to evaluate the safety and prophylactic effect of variceal treatment with endoscopic procedures and balloon-occluded retrograde transvenous obliteration (BRTO) in advanced LC.

\section{MATERIALS AND METHODS}

The study included 51 patients with CP-C end-stage LC who underwent endoscopic procedures or BRTO for esophageal/gastric varices between April 1995 and December 2017. The characteristics of the study population are shown in Table 1. There were 40 men and 11 women, with a mean age of $60.4 \pm 10.7$ years. The etiology of $L C$ was hepatitis $C$ virus infection in 19 patients, hepatitis B virus infection in 6 patients, alcohol injury in 18 patients, and others in 8 patients. The Child-Pugh scores (CPS) in each etiology were 10 in 35 patients, 11 in 8 patients, 12 in 3 patients, 13 in 3 patients, and 14 in 2 patients, respectively. The purpose of treatment was bleeding in 31 patients, prophylaxis in 20 patients. The treatment procedure was endoscopic variceal ligation (EVL) in 22 patients, endoscopic injection sclerotherapy (EIS) in 17 patients (5\% ethanolamine oleato injection in 10 patients and a-cyanoacrylate monomer injection in 7 patients) and BRTO in 12 patients. The survival time was less than 1 year in 21 patients, more than 1 year in 22 patients, and unknown in 8 patients.

Clinical variables were analyzed in three categories: (1) the effect of all procedures on patient outcomes as assessed by the overall survival (OS) rates in all patients; (2) respective outcomes in patients according to the type of procedures (endoscopic treatments and BRTO) as assessed by the OS rates in the endoscopic treatment group ( $n=39)$ and the BRTO group $(n=12)$ in all pa-

Table 1. Clinical characteristics of the study population

\begin{tabular}{|c|c|}
\hline & Values \\
\hline Number of patients & 51 \\
\hline Gender (male/female) & $40 / 11$ \\
\hline Age (years) & $60.4 \pm 10.7(28-79)$ \\
\hline \multicolumn{2}{|l|}{ Etiology (n, \%) } \\
\hline $\mathrm{HCV}$ & $19(37.2)$ \\
\hline HBV & $6(11.8)$ \\
\hline Alcohol & $18(35.3)$ \\
\hline Others & $8(15.7)$ \\
\hline \multicolumn{2}{|l|}{ Child-Pugh Score (n, \%) } \\
\hline 10 point & $35(68.7)$ \\
\hline 11 point & $8(15.7)$ \\
\hline 12 point & $3(5.9)$ \\
\hline 13 point & $3(5.9)$ \\
\hline 14 point & $2(3.8)$ \\
\hline \multicolumn{2}{|c|}{ Purpose of treatment $(n, \%)$} \\
\hline Bleeding & $31(60.8)$ \\
\hline Prophylactic & $20(39.2)$ \\
\hline \multicolumn{2}{|c|}{ Method of treatment $(n, \%)$} \\
\hline EVL & $22(43.1)$ \\
\hline EIS & $17(33.3)$ \\
\hline BRTO & $12(23.6)$ \\
\hline \multicolumn{2}{|l|}{ Survival period $(n, \%)$} \\
\hline Less than one year & $21(41.2)$ \\
\hline Over one year & $22(43.1)$ \\
\hline Unknown & $8(15.7)$ \\
\hline
\end{tabular}

Values are presented as mean \pm standard deviation (range) or $n(\%)$ unless otherwise indicated.

$H C V$, hepatitis C virus; HBV, hepatitis B virus; EVL, esophageal variceal ligation; EIS, endoscopic injection sclerotherapy; BRTO, balloon-occluded retrograde transvenous obliteration. 
Keiji Yokoyama, et al. Invasive treatment for varices in Child-Pugh class C

tients, and by changes in CPS; and, (3) factors contributing to death within 1 year of treatment.

Our research protocol was approved by the Ethics Committee of Fukuoka University Hospital (Approval No.16-1-16).

Statistical analyses were performed using JMP version 10.0 (SAS Institute, Cary, NC, USA). The methods of changes in each parameter and comparison between groups included Wilcoxon signedrank test, the $t$-test and chi-square test. The cumulative survival curves were calculated according to the Kaplan-Meier method, a log-rank test was used for the comparison between each curve. $P$-values less than 0.05 were considered significant.

\section{RESULTS}

The OS rates in all 51 patients at 1, 2, 3 and 5 years were

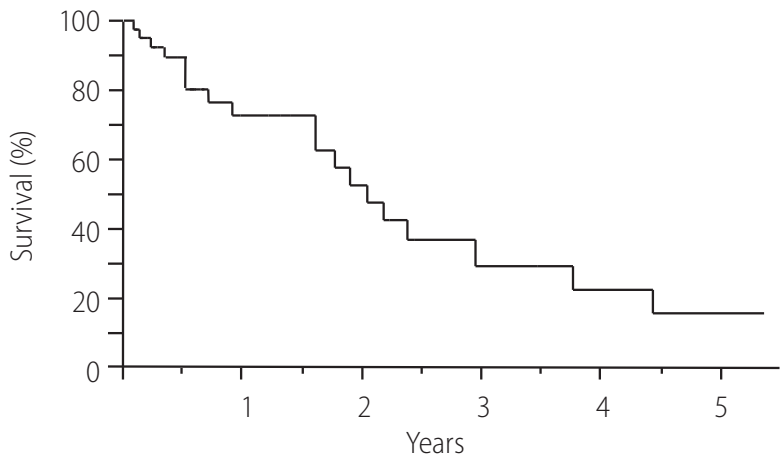

Figure 1. The overall survival (OS) rates in all patients $(n=51)$. The OS rates in all 51 patients at 1, 2, 3 and 5 years were $72.6 \%, 50.1 \%, 30.2 \%$ and $15.1 \%$, respectively.

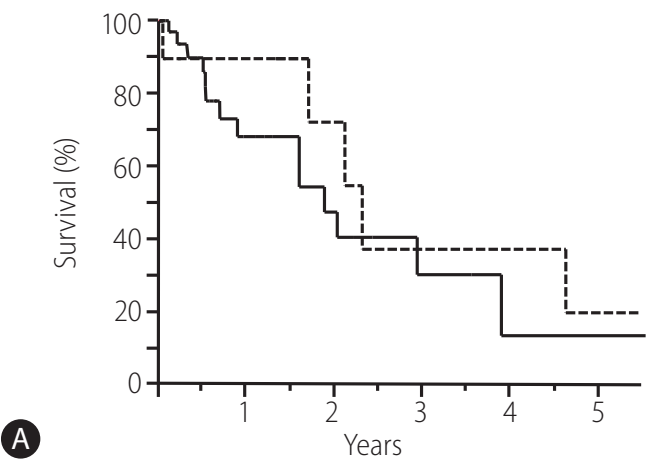

$72.6 \%, 50.1 \%, 30.2 \%$ and $15.1 \%$, respectively (Fig. 1). The characteristics of the endoscopic treatment group and the BRTO group were shown in Table 2. The OS rates at 1, 2, 3 and 5 years were $67.6 \%, 43.9 \%, 28.2 \%$ and $14.1 \%$ in the endoscopic treatment group, respectively (Fig. 2A, solid line). There was no significant difference in OS between 22 patients treated with EVL and 17 patients treated with EIS ( $P=0.262$, figure not shown). The OS rate at $1,2,3$ and 5 years were $90.0 \%, 72.0 \%, 36.0 \%$ and $18.0 \%$ in the BRTO group, respectively (Fig. 2A, dotted line). There were no

Table 2. Background characteristics of the endoscopic group and the BRTO group

\begin{tabular}{lccc}
\hline & $\begin{array}{c}\text { Endoscopy } \\
\text { (EVL or EIS) }\end{array}$ & BRTO & P-value \\
\hline $\begin{array}{l}\text { Number of patients } \\
\text { Gender } \\
\text { Male/Female }\end{array}$ & 39 & 12 & \\
\hline $\begin{array}{l}\text { Age (years) } \\
\text { Etiology } \\
\text { Viral hepatitis/Non-viral }\end{array}$ & $60.3 \pm 10.3$ & $60.7 \pm 9.4$ & 0.926 \\
$\begin{array}{l}\text { Child-Pugh Score } \\
\text { 10 point/Over 11 point }\end{array}$ & $26 / 19$ & $5 / 7$ & 0.560 \\
$\begin{array}{l}\text { Purpose of treatment } \\
\text { Bleeding/Prophylaxis }\end{array}$ & $26 / 13$ & $9 / 3$ & 0.586 \\
\hline $\begin{array}{c}\text { Coexist of HCC } \\
\text { Present/Absent }\end{array}$ & $16 / 23$ & $5 / 7$ & 0.121 \\
\hline
\end{tabular}

Values are presented as mean \pm standard deviation unless otherwise indicated.

BRTO, balloon-occluded retrograde transvenous obliteration; EVL, esophageal variceal ligation; EIS, endoscopic injection sclerotherapy; HCC, hepatocellular carcinoma.

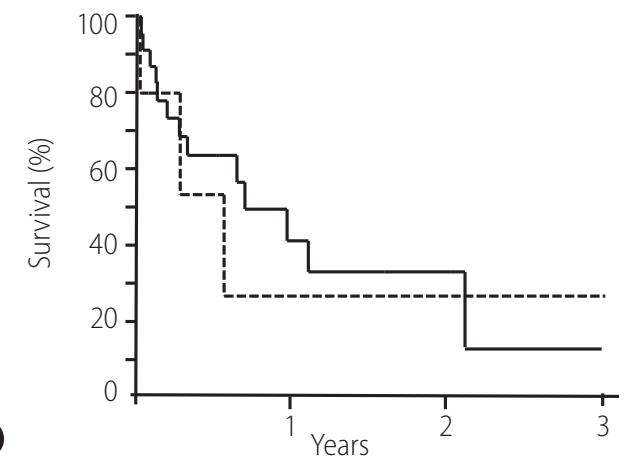

Figure 2. In a comparison of the overall survival (OS) rates in the endoscopic treatment group and in the BRTO group. (A) The OS rates in the endoscopic treatment group $(n=39)$ and in the BRTO group $(n=12)$. The OS rates at 1, 2, 3 and 5 years were $67.6 \%, 43.9 \%, 28.2 \%$ and $14.1 \%$ in the endoscopic treatment group (solid line) and $90.0 \%, 72.0 \%, 36.0 \%$ and $18.0 \%$ in the BRTO group (dotted line), respectively. There were no significant differences in the survival rates between the groups. (B) The OS rates in patients with bleeding varices in the endoscopic treatment group ( $n=26$ ) and the BRTO group ( $n=5)$. The OS rates in patients with bleeding variceal treatment at 1, 2 and 3 years were $41.2 \%, 33.6 \%$ and $13.2 \%$ in the endoscopic treatment group (solid line) and $26.7 \%, 26.7 \%$ and $26.7 \%$ in the BRTO group (dotted line), respectively. There were no significant differences in the survival rates between the groups. BRTO, balloon-occluded retrograde transvenous obliteration. 
T-bil (mg/dL)
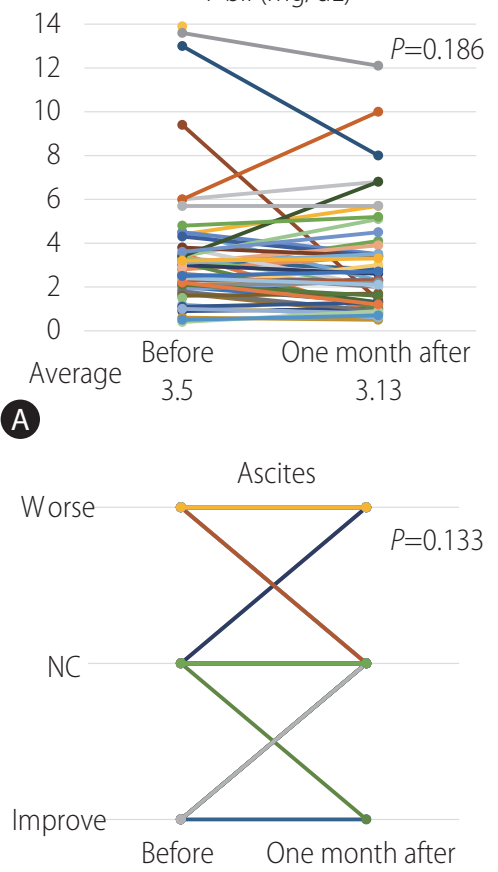

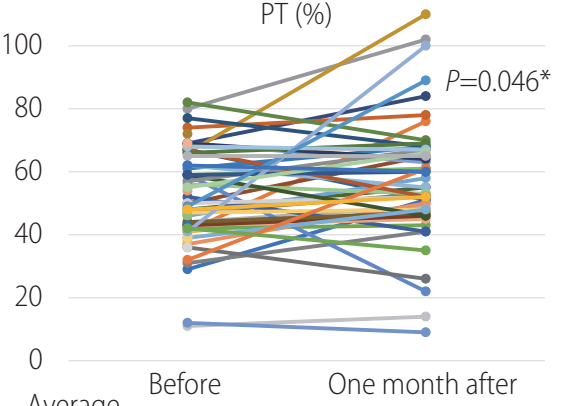

B

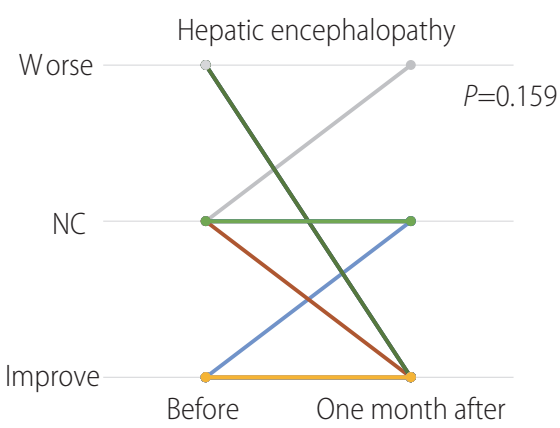

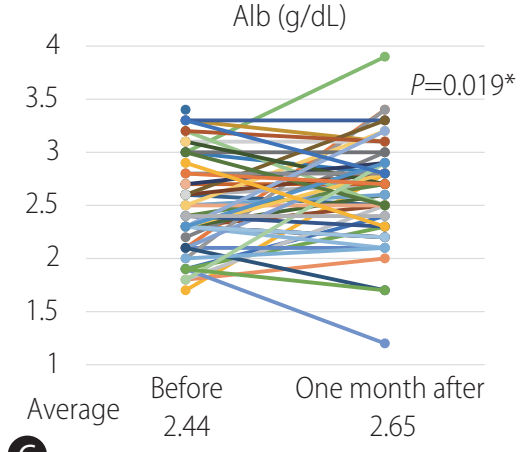

C

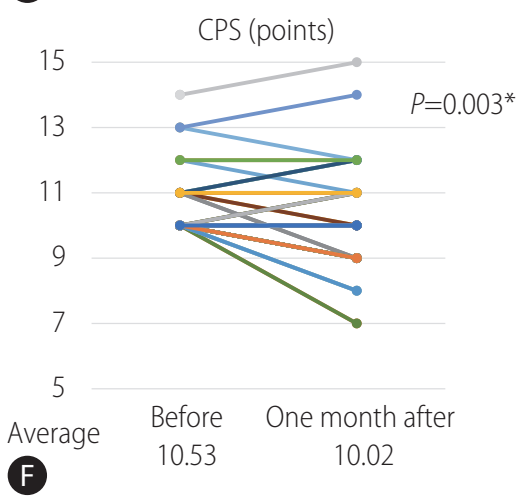

(D)

E

Figure 3. Changes in CPS and their associated parameters from before treatment to one month after treatment for varices ( $n=45)$. (A) T-bil. (B) PT activity. (C) Alb. (D) Ascites. (E) Hepatic encephalopathy. (F) CPS. T-bil, total bilirubin; PT, prothrombin activity; Alb, albumin; NC, no change; CPS, ChildPugh Score. " $P<0.05$ was considered statistically significant.
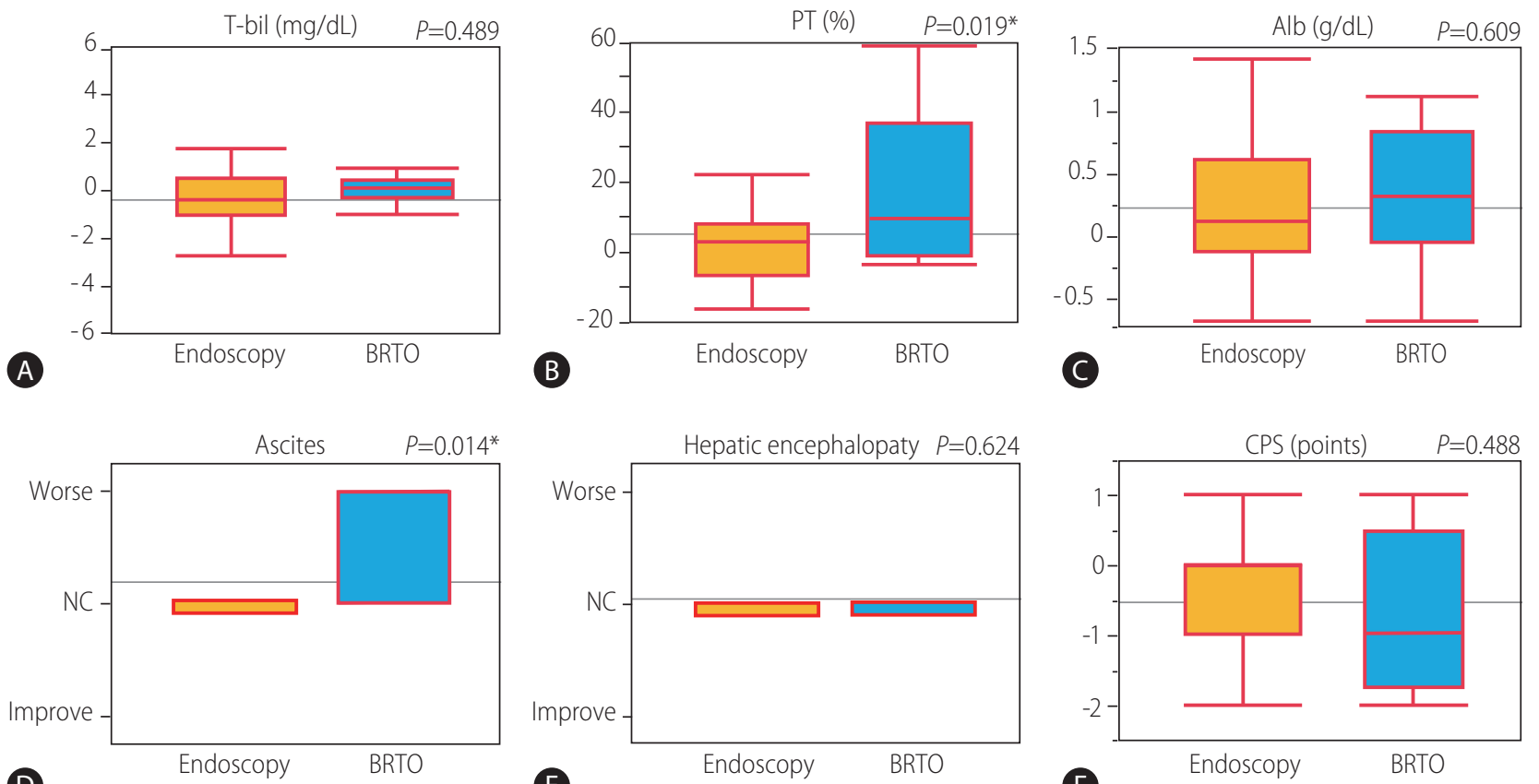

Figure 4. In a comparison of the mean change ratio in CPS and the associated parameters from before treatment to one month after variceal treatment between the endoscopic treatment group ( $n=37)$ and the BRTO group ( $n=8)$. (A) T-bil. (B) PT activity. (C) Alb. (D) Ascites. (E) Hepatic encephalopathy. (F) CPS. T-bil, total bilirubin; PT, prothrombin activity; Alb, albumin; BRTO, balloon-occluded retrograde transvenous obliteration; NC, no change; CPS, Child-Pugh Score. " $P<0.05$ was considered statistically significant. 
Keiji Yokoyama, et al. Invasive treatment for varices in Child-Pugh class C

significant differences in the survival rates between the groups. The OS rates in patients with bleeding variceal treatment at 1, 2 and 3 years were $41.2 \%, 33.6 \%$ (Fig. 2B, solid line), and 13.2\% in the endoscopic treatment group and $26.7 \%, 26.7 \%$ and $26.7 \%$ in the BRTO group, respectively (Fig. 2B, dotted line).

45 of 51 patients with variceal treatment were able to follow-up one month after treatment. Changes in CPS and their associated parameter from before treatment to 1 month after treatment were shown in Figure 3. The average of serum prothrombin activity and serum albumin levels was significantly improved $(P=0.046$ and 0.019), and the average of CPS was significantly improved from 10.53 to $10.02(P=0.003)$ from before treatment to 1 month after

Table 3. Univariate analysis of factors contributing to death within one year of treatment

\begin{tabular}{|c|c|c|c|}
\hline & \multicolumn{3}{|c|}{ Survival period } \\
\hline & $<$ One year & $\geq 0$ ne year & $P$-value \\
\hline Number of patients & 22 & 21 & \\
\hline Age (years) & $61.0 \pm 1.99$ & $58.8 \pm 2.63$ & 0.499 \\
\hline $\begin{array}{l}\text { Gender } \\
\text { Male/Female }\end{array}$ & $19 / 3$ & $14 / 7$ & 0.126 \\
\hline $\begin{array}{l}\text { Etiology } \\
\text { Viral/Non-viral }\end{array}$ & $10 / 12$ & 10/11 & 0.887 \\
\hline Child-Pugh score & $11.1 \pm 0.19$ & $10.1 \pm 0.06$ & $0.002^{*}$ \\
\hline PT (\%) & $48.2 \pm 3.75$ & $53.0 \pm 2.93$ & 0.316 \\
\hline Alb (g/dL) & $2.47 \pm 0.11$ & $2.53 \pm 0.10$ & 0.707 \\
\hline T-bil (mg/dL) & $4.30 \pm 0.65$ & $2.87 \pm 0.66$ & 0.132 \\
\hline $\begin{array}{l}\text { Ascites } \\
\text { Present/Absent }\end{array}$ & $20 / 2$ & $19 / 2$ & 0.961 \\
\hline $\begin{array}{l}\text { Hepatic encephalopathy } \\
\text { Present/Absent }\end{array}$ & $6 / 16$ & $2 / 19$ & 0.135 \\
\hline $\begin{array}{l}\text { Methods of treatment } \\
\text { Endoscopic/BRTO }\end{array}$ & $17 / 5$ & $14 / 7$ & 0.438 \\
\hline $\begin{array}{l}\text { Variceal bleeding } \\
\text { Present/Absent }\end{array}$ & $16 / 6$ & $9 / 12$ & $0.047^{*}$ \\
\hline $\begin{array}{l}\text { HCC } \\
\text { Present/Absent }\end{array}$ & $11 / 11$ & $8 / 13$ & 0.432 \\
\hline $\begin{array}{l}\text { T-bil (mg/dL) } \\
\text { Less than 4/Over } 4\end{array}$ & $13 / 9$ & $19 / 2$ & $0.018^{*}$ \\
\hline $\begin{array}{l}\text { Child-Pugh Score } \\
10 \text { point/Over } 11 \text { point }\end{array}$ & $11 / 11$ & $19 / 2$ & $0.004^{*}$ \\
\hline
\end{tabular}

Values are presented as mean \pm standard deviation unless otherwise indicated.

PT, prothrombin activity; Alb, albumin; T-bil, total bilirubin; BRTO, balloonoccluded retrograde transvenous obliteration; HCC, hepatocellular carcinoma.

${ }^{*} P<0.05$ was considered statistically significant. treatment. The comparison of the mean change ratio in CPS and their associated parameter from before treatment to 1 month after treatment between the endoscopic treatment group $(n=37)$ and the BRTO group $(n=8)$ were shown in Figure 4 . The average of serum prothrombin activity was significantly improved in the BRTO group ( $P=0.019)$. Meanwhile, ascites was significantly to become worse in the BRTO group $(P=0.014)$. Comprehensively, there was no significant difference of the mean change ratio in CPS between the groups.

As shown in Table 3, univariate analysis of factors contributing to death within 1 year of treatment identified three significant variables: the presence of bleeding varices, high CPS $(\geq 11)$ and high serum total bilirubin levels ( $\geq 4.0 \mathrm{mg} / \mathrm{dL}$ ). The presence of hepatocellular carcinoma (HCC) was not significantly associated with death within 1 year of treatment.

\section{DISCUSSION}

A previous important systematic review reported that any studies regarding predictors of death should include the CPS and age in patients with decompensated cirrhosis. ${ }^{9}$ This large systematic review demonstrated that the OS rates at 1, 2, 3 and 5 years were approximately $60 \%, 45 \%, 35 \%$ and $25 \%$ in a large cohort of decompensated LC, respectively. Moreover, the OS rates were $45 \%$ at 1 year and $38 \%$ at 2 years in patients with CP-C end-stage LC. According to a recent report in Japan, the cumulative 2-year survival rates of $\mathrm{CP}-\mathrm{C}$ patients in the liver transplantation registry were $38.1 \%{ }^{10}$ Compared with these reports, the OS rates after treatment in all patients, and in each treatment group were higher than those in the present study. A reason for the better survival outcome may be associated with the relatively large proportion of patients with a CPS of 10. In addition, CPS was significantly decreased after variceal treatment in the present study. It can be explained by that liver function could be improved by multidisciplinary treatment for cirrhosis after invasive treatment, even in patients with CP-C end-stage LC.

BRTO was first described by Kanagawa et al. in 1996 as a treatment for isolated gastric varices. ${ }^{11}$ Although there are concerns about the adverse effects of BRTO, including renal dysfunction by hemolysis, worsening of esophageal varices, and increased ascites, it has been reported that BRTO contributed to improve survival. $^{12-18}$ In the comparison of the mean change ratio in CPS and their each parameter from before treatment to 1 month after treatment between the endoscopic treatment group and the BRTO 
group, the average of prothrombin activity was significantly improved in the BRTO group, meanwhile ascites was significantly worsen in the BRTO group. Comprehensively, there was no significant difference of the mean change ratio in CPS between the groups. Previous studies have shown that occlusion of a large portosystemic shunt by BRTO increases the effective portal blood flow into the liver and improves hepatic functional reserve ${ }^{19-23}$ which is likely to contribute to improve survival rates in the present study. In contrast, BRTO has been linked to the risk of portal vein thrombosis or impaired intrahepatic vascular perfusion by outflow of ethanolamine oleate to portal veins or the lung, subsequent hepatic failure, decreased respiratory function, ${ }^{24}$ and balloon rupture..$^{25}$ Therefore, for BRTO to be implemented safely and effectively, it is necessary to learn the appropriate technical skills. Recently, form-BRTO ${ }^{26,27}$ or combination therapy with BRTO and partial splenic embolization ${ }^{28}$ was found to safely improve hepatic function by reducing the use of $5 \%$ ethanolamine oleate.

In the present study, there was no significant difference in the survival rates between patients treated with EVL versus EIS. The mean dose of $5 \%$ ethanolamine oleate used for EIS was $9.1 \pm 5.80 \mathrm{~mL}$, which was set at approximately $0.2 \mathrm{~mL} / \mathrm{kg}$, a relatively low dose considering hepatic functional reserve. This dose setting could be responsible for the lack of difference in the survival rates between $\mathrm{EVL}$ and EIS despite the less invasive nature of EVL.

In the present study, the presence of HCC was not a significant factor contributing to death within 1 year of treatment. Because none of the patients with HCC had severe portal invasion of portal vein invasion of the second branches or higher, it is possible that hepatic failure, rather than cancer progression, was the main cause of death in most cases.

Another finding was that three variables such as the presence of bleeding varices, higher CPS (11 or higher) and higher serum total bilirubin levels $(4.0 \mathrm{mg} / \mathrm{dL}$ or higher) were significantly related to an increased risk of death within 1 year of treatment. However, in patients with bleeding, it is difficult to determine whether the cause of early death is the invasiveness of treatment itself or the reduced hepatic functional reserve due to bleeding and ischemia. The present results suggested that CP-C end-stage LC patients with esophageal/gastric varices and/or hepatic encephalopathy which required treatment, together with advanced jaundice (serum total bilirubin levels of $4.0 \mathrm{mg} / \mathrm{dL}$ or higher) and/or high CPS (11 or higher), have a poor prognosis after treatment. Clinicians should be aware of these factors to enable them to inform patients and to perform treatment in an optimal manner. Complications of EIS or BRTO can easily lead to hepatic failure, therefore continuous efforts are necessary to perform procedures skillfully and to establish a safe and effective standard treatment.

A limitation of the present study design is not being a comparative/randomized trial. Various patients with heterogenous background (etiology of cirrhosis, purpose of treatment, history of variceal bleeding) were enrolled. Therefore it is difficult for effective comparison of clinical usefulness between endoscopic procedure and BRTO. Furthermore, in this study, only univariate analysis was performed, not multivariate analysis because of the small sample size. It is necessary to analyze data from a larger population in future studies.

In conclusion, there is a conflicting research about invasive treatments such as endoscopic procedures and BRTO for patients with CP-C end-stage LC. The findings of this study suggested that, among patients with $\mathrm{CP}-\mathrm{C}$ end-stage $\mathrm{LC}$, those with lower CPS (10 or lower) and lower serum total bilirubin levels $(4.0 \mathrm{mg} / \mathrm{dL}$ or lower) did not have a worse prognosis after invasive treatment.

\section{Authors' contribution}

K.Y. designed and carried out the study; All authors analyzed the data; K.Y. wrote the paper.

\section{Acknowledgements}

The authors gratefully acknowledge Prof. Kengo Yoshimitsu, Chairman in the Department of Radiology, Fukuoka University, and Associate Prof. Hideyuki Higashihara for performing BRTO procedures in the Department of Radiology, Fukuoka University Chikushi Hospital.

\section{Conflicts of Interest}

The authors declare that there is no conflict of interests regarding the publication of this paper.

\section{REFERENCES}

1. Orrego H, Blendis LM, Crossley IR, Medline A, Macdonald A, Ritchie $S$, et al. Correlation of intrahepatic pressure with collagen in the Disse space and hepatomegaly in humans and in the rat. Gastroenterology 1981;80:546-556.

2. Blendis LM, Orrego H, Crossley IR, Blake JE, Medline A, Isreal Y. The role of hepatocyte enlargement in hepatic pressure in cirrhotic and noncirrhotic alcoholic liver disease. Hepatology 1982;2:539-546.

3. Grossman HJ, Grossman VL, Bhathal PS. The effect of hepatocyte enlargement on the hemodynamic characteristics of the isolated perfused rat liver preparation. Hepatology 1998;27:446-451. 
4. Bhathal PS, Grossman HJ. Reduction of the increased portal vascular resistance of the isolated perfused cirrhotic rat liver by vasodilators. J Hepatol 1985;1:325-337.

5. Chao Y, Lin HC, Lee FY, Wang SS, Tsai YT, Hsia HC, et al. Hepatic hemodynamic features in patients with esophageal or gastric varices. J Hepatol 1993;19:85-89.

6. Hasumi A, Aoki H, Shimazu M, Kawata S, Yoshimatsu Y. Causative relationship between a hyperdynamic state due to increased $A-V$ anastomoses in the gastric wall and AGML in patients with liver cirrhosis. J Gastroenterol Hepatol 1989;4 Suppl 1:143-145.

7. Masaki M, Obara K, Suzuki S, Orikasa K, Mitsuhashi H, Iwasaki K, et al. The destructive effects of sclerosant ethanolamine oleate on mammalian vessel endothelium. Gastroenterol Jpn 1990;25:230-235.

8. Iwase H, Kusugami K. Symposium on endoscopic hemostasis in gastric diseases. 2. Choice of endoscopic therapy for esophagogastric varices: aiming for safer and more effective therapy. Intern Med 1997;36:128-129.

9. D’Amico G, Garcia-Tsao G, Pagliaro L. Natural history and prognostic indicators of survival in cirrhosis: a systematic review of 118 studies. J Hepatol 2006;44:217-231.

10. Genda T, Ichida T, Sakisaka S, Tanaka E, Mochida S, Ueno Y, et al. Survival in patients with Child-Pugh class C cirrhosis: analysis of the liver transplant registry in Japan. Hepatol Res 2017;47:1155-1164.

11. Kanagawa H, Mima S, Kouyama H, Gotoh K, Uchida T, Okuda K. Treatment of gastric fundal varices by balloon-occluded retrograde transvenous obliteration. J Gastroenterol Hepatol 1996;11:51-58.

12. Park JK, Saab S, Kee ST, Busuttil RW, Kim HJ, Durazo F, et al. Balloon-Occluded Retrograde Transvenous Obliteration (BRTO) for treatment of gastric varices: review and meta-analysis. Dig Dis Sci 2015;60:1543-1553.

13. Numata K, Tanaka K, Kiba T, Saito S, Shirato K, Kitamura T, et al. Use of balloon-occluded retrograde transvenous obliteration with ethanolamine oleate for the treatment of hepatic encephalopathy in a cirrhotic patient with a large spontaneous splenorenal shunt. J Gastroenterol 1998;33:424-427.

14. Kawanaka H, Ohta M, Hashizume M, Tomikawa M, Higashi H, Kishihara $F$, et al. Portosystemic encephalopathy treated with balloonoccluded retrograde transvenous obliteration. Am J Gastroenterol 1995;90:508-510.

15. Saad WE. Balloon-occluded retrograde transvenous obliteration of gastric varices: concept, basic techniques, and outcomes. Semin Intervent Radiol 2012;29:118-128.

16. Garcia-Pagán JC, Barrufet M, Cardenas A, Escorsell A. Management of gastric varices. Clin Gastroenterol Hepatol 2014;12:919-928.e1; quiz e51-e52.

17. Tanihata H, Minamiguchi H, Sato M, Kawai N, Sonomura T, Takasaka I, et al. Changes in portal systemic pressure gradient after balloon-occluded retrograde transvenous obliteration of gastric varices and aggravation of esophageal varices. Cardiovasc Intervent Radiol 2009;32:1209-1216.

18. Choi YS, Lee JH, Sinn DH, Song YB, Gwak GY, Choi MS, et al. Effect of balloon-occluded retrograde transvenous obliteration on the natural history of coexisting esophageal varices. J Clin Gastroenterol 2008:42:974-979.

19. Fukuda T, Hirota S, Sugimura K. Long-term results of balloonoccluded retrograde transvenous obliteration for the treatment of gastric varices and hepatic encephalopathy. J Vasc Interv Radiol 2001;12:327-336.

20. Jang SY, Kim GH, Park SY, Cho CM, Tak WY, Kim JH, et al. Clinical outcomes of balloon-occluded retrograde transvenous obliteration for the treatment of gastric variceal hemorrhage in Korean patients with liver cirrhosis: a retrospective multicenter study. Clin Mol Hepatol 2012;18:368-374.

21. Miyamoto Y, Oho K, Kumamoto M, Toyonaga A, Sata M. Balloonoccluded retrograde transvenous obliteration improves liver function in patients with cirrhosis and portal hypertension. J Gastroenterol Hepatol 2003;18:934-942.

22. Akahane T, Iwasaki T, Kobayashi N, Tanabe N, Takahashi N, Gama $\mathrm{H}$, et al. Changes in liver function parameters after occlusion of gastrorenal shunts with balloon-occluded retrograde transvenous obliteration. Am J Gastroenterol 1997;92:1026-1030.

23. Uehara H, Akahoshi T, Tomikawa M, Kinjo N, Hashimoto N, Nagao $Y$, et al. Prediction of improved liver function after balloon-occluded retrograde transvenous obliteration: relation to hepatic vein pressure gradient. J Gastroenterol Hepatol 2012;27:137-141.

24. Park SJ, Chung JW, Kim HC, Jae HJ, Park JH. The prevalence, risk factors, and clinical outcome of balloon rupture in balloon-occluded retrograde transvenous obliteration of gastric varices. J Vasc Interv Radiol 2010;21:503-507.

25. Arai H, Abe T, Takayama H, Toyoda M, Mori K, Ueno T, et al. Respiratory effects of balloon occluded retrograde transvenous obliteration of gastric varices: a prospective controlled study. J Gastroenterol Hepatol 2011;26:1389-1394.

26. Choi SY, Won JY, Kim KA, Lee DY, Lee KH. Foam sclerotherapy using polidocanol for balloon-occluded retrograde transvenous obliteration (BRTO). Eur Radiol 2011;21:122-129.

27. Clements W, Cavanagh K, Ali F, Kavnoudias H, Kemp W, Roberts S, et al. Variant treatment for gastric varices with polidocanol foam using balloon-occluded retrograde transvenous obliteration: a pilot study. J Med Imaging Radiat Oncol 2012;56:599-605.

28. Yoshida H, Mamada Y, Taniai N, Yamamoto K, Kaneko M, Kawano $Y$, et al. Long-term results of partial splenic artery embolization as supplemental treatment for portal-systemic encephalopathy. Am J Gastroenterol 2005;100:43-47. 